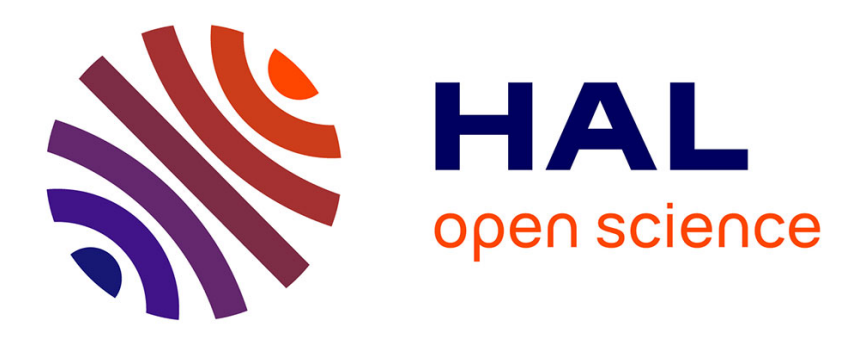

\title{
Notes sur la piraterie moderne en Méditerranée Sud-Est asiatique
}

\author{
Daniel Perret
}

\section{To cite this version:}

Daniel Perret. Notes sur la piraterie moderne en Méditerranée Sud-Est asiatique. Archipel, 1998, 56, pp.121-144. 10.3406/arch.1998.3480 . halshs-01883823

\section{HAL Id: halshs-01883823 \\ https://shs.hal.science/halshs-01883823}

Submitted on 28 Sep 2018

HAL is a multi-disciplinary open access archive for the deposit and dissemination of scientific research documents, whether they are published or not. The documents may come from teaching and research institutions in France or abroad, or from public or private research centers.
L'archive ouverte pluridisciplinaire HAL, est destinée au dépôt et à la diffusion de documents scientifiques de niveau recherche, publiés ou non, émanant des établissements d'enseignement et de recherche français ou étrangers, des laboratoires publics ou privés. 


\section{Notes sur la piraterie moderne en Méditerranée Sud-Est asiatique} Daniel Perret

\section{Citer ce document / Cite this document :}

Perret Daniel. Notes sur la piraterie moderne en Méditerranée Sud-Est asiatique. In: Archipel, volume 56, 1998. L'horizon nousantarien. Mélanges en hommage à Denys Lombard (Volume I) pp. 121-144;

doi : https://doi.org/10.3406/arch.1998.3480

https://www.persee.fr/doc/arch_0044-8613_1998_num_56_1_3480

Fichier pdf généré le 21/04/2018 


\section{Daniel PERRET}

\section{Notes sur la piraterie moderne en Méditerranée Sud-Est asiatique}

Dans un article paru il y a près de vingt ans dans cette même revue(1), Denys Lombard analysait la piraterie dans le monde malais de la première moitié du XIX ${ }^{\mathrm{e}}$ siècle comme l'une des composantes des conflits opposant les populations locales aux puissances coloniales. Il la présentait comme «la dernière résurgence de ces réseaux asiatiques, essentiellement musulmans, accessoirement chinois » dont il s'est toujours attaché à démontrer l'impor-

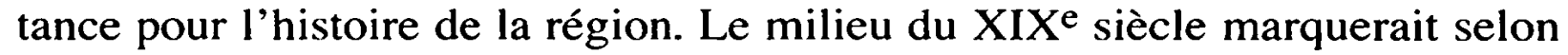
lui la fin d'une époque, une rupture. D'agresseur le pirate deviendrait résistant face au mouvement vainqueur des Occidentaux qui, en reprenant progressivement le contrôle des mers, marginalisent et étouffent ses réseaux. «La piraterie est aux abois»(2) au cours des dernières décennies du XIX siècle.

Un siècle plus tard, la présence menaçante du pirate semble refaire surface dans la région. Sur le plan mondial au cours de ces dernières années, la majorité des actes de piraterie maritime ont été commis en Méditerranée Sud-Est asiatique et leur forte augmentation depuis 1995 inquiète organisations internationales, gouvernements locaux et tous les agents économiques

1. Denys Lombard, «Regard nouveau sur les “pirates malais”, lère moitié du XIX" siècle», Archipel 18, $1979: 231-250$.

2. Ibid. : 232. 
concernés qui la perçoivent comme une réelle menace pour les activités maritimes.

Comment se présente la carte de la piraterie moderne en Méditerranée Sud-Est asiatique? En quoi les stratégies et les éventuels réseaux de la piraterie moderne dans la région diffèrent-ils de ceux de la piraterie de la première moitié du $\mathrm{XIX}^{\mathrm{e}}$ siècle ? Les formes de lutte contre la piraterie maritime sont-elles fondamentalement différentes de celles pratiquées il y a un siècle et demi ? Pour tenter d'apporter des éléments de réponse à ces questions, nous ferons appel essentiellement à deux types de sources : d'une part, les rapports annuels (de 1991 à mars 1998) du Centre de renseignement régional sur la piraterie basé à Kuala Lumpur, la presse quotidienne malaisienne (New Straits Times, Berita Harian, Utusan Malaysia, Harian Watan, The Star) depuis mars 1995, d'autre part.

\section{Définition et pratique moderne de la piraterie}

En 1982, la Convention des Nations Unies sur le droit de la mer (UNCLOS) définissait légalement l'acte de piraterie comme une attaque perpétrée à des fins privées sur un bateau en haute mer, avec usage de la violence, détention illégale de personnes ou de propriétés, ou vol et destruction de biens. L'UNCLOS considère comme «haute mer» tout l'espace non compris dans la zone économique exclusive (200 miles nautiques de l'île la plus éloignée de la côte), dans les eaux territoriales ( 12 miles de l'île la plus éloignée de la côte) ou dans les eaux intérieures d'un État. De son côté, dans un but plus pratique que légal, le Bureau maritime international (IMB) définit comme acte de piraterie tout abordage dans l'intention de commettre un vol ou autre crime avec éventuellement usage de la force pour parvenir à ses fins. Cette définition élargie, qui recouvre aussi bien les vols et tentatives de vols dans les ports que les attaques et tentatives d'attaques à main armée en haute mer, n'est pas toujours acceptée par les autorités locales. Ainsi la police maritime malaisienne considère que les attaques commises dans les eaux territoriales malaisiennes sont du brigandage maritime et non de la piraterie (3). De même, elle enregistre les attaques contre des bateaux à quai comme de simples vols (4). Ces divergences d'interprétation ne sont pas sans importance, au moins sur le plan psychologique. Les autorités malaisiennes ont pu en effet affirmer que leurs eaux territoriales étaient exemptes de piraterie en

3. New Straits Times, 23.5.96; New Sunday Times, 6.7.97.

4. New Sunday Times, 6.7.97. 
1996, les cinq attaques rapportées par l'IMB ayant eu lieu sur des bateaux à quai (5). Nous retiendrons pour notre part la définition du Bureau maritime international puisque les statistiques présentées émanent de cet organisme.

Les informations concernant les attaques de pirates sont généralement très fragmentaires. Elles émanent, soit du Centre régional de renseignement sur la piraterie, soit des autorités policières, et sont parfois relayées par la presse locale. Elles nous éclairent toutefois sur la localisation des attaques, les stratégies des pirates, leur nationalité ainsi que sur le butin prélevé. Nous donnons ici par ordre chronologique les informations les plus éclairantes tirées de ces sources depuis la mise en place du Centre régional en 1992.

\section{Année 1994(6)}

En janvier 1994, le Jui Ho est attaqué dans les eaux vietnamiennes par une vingtaine de pirates lourdement armés. L'équipage une fois maîtrisé, le navire fait route vers une destination inconnue où la cargaison est déchargée. Le détournement aura duré quatre jours. Le même mois le Alicia Star est détourné par des pirates également fortement armés dans la zone Hong Kong - Luzon - Hainan. Le navire est conduit au port chinois de Shanwei où sa cargaison de cigarettes d'une valeur de 5 millions de dollars US est déchargée. Deux mois plus tard, le Tequila, qui fait route vers Keelung (Taiwan) avec une cargaison de voitures d'occasion, est investi au large de Hong Kong par cinq pirates qui le détournent vers Wailing dao, où sa cargaison est déchargée.

\section{Année 1995(7)}

En janvier 1995, le Kafu Maru est attaqué dans le port de Subic, près de Manille, par dix garde-côtes qui subtilisent 380 caisses d'alcool et de cigarettes d'importation. À la même époque le Troianu est attaqué alors qu'il décharge sa cargaison dans le port de Belawan (près de Medan, Sumatra). Les assaillants repartent avec 21 bidons de peinture. Deux abordages se déroulent au même endroit dans les semaines qui suivent. Les pirates repartiront les mains vides dans le premier cas, avec un rouleau de cordage et sept bidons de peinture dans le second cas. À l'ancre devant le port de Sandakan (Sabah) durant une tempête ce même mois de janvier, le Norbulk Pine est investi par des pirates qui repartent avec 600 litres de peinture. Mi-février, en

\footnotetext{
5. Ibid.

6. International Maritime Bureau, Piracy Report 1994.

7. International Maritime Bureau, Pircuy Report 1995 (sauf autre indication).
} 
Mer de Chine méridionale, des pirates prennent le contrôle du Lucky Trader No. 1 en provenance de Singapour. Quelques heures plus tard, 7000 cartons de cigarettes, des biens personnels et des équipements de télécommunication d'une valeur de 100000 ringgit sont transférés sur une seconde embarcation (8). En février, des pirates investissent le $L P G$ Norgas à l'ancre devant le port d'Anyer (Java Ouest) et repartent avec des pièces détachées de moteurs. Une attaque similaire se produira trois mois plus tard. En mars, six pirates munis d'armes blanches investissent le Bunga Melor Satu dans le port de Jakarta, volent des pièces détachées et s'enfuient à bord d'un perahu. En avril, le Calatagan, à l'ancre devant le port de Surabaya, est investi par quatre pirates munis d'armes blanches qui s'enfuient avec 295 litres de peinture et un canot de sauvetage. Toujours en avril, le Vietxo 8, transportant 11 voitures de Hong Kong à Cua la (Viêt Nam), est intercepté à cinq miles au large des eaux de Hong Kong par la marine chinoise. Toutes les tentatives pour entrer alors en contact avec le navire restent vaines. En mai, à trois miles du phare de Horsburgh dans le Détroit de Singapour, six pirates tirent une trentaine de coups de feu sur un chalutier avant de l'aborder pour demander une rançon de 30000 dollars US. L'équipage ne disposant pas de cette somme, les assaillants mettent le navire à sac, s'emparent d'espèces, d'équipements et du produit de la pêche avant d'obliger le capitaine à contacter un navire proche pour obtenir la rançon demandée. Les pirates s'enfuiront finalement avec 15000 dollars US. En juin, un ferry rapide faisant la liaison entre Macau et Hong Kong avec à son bord 129 passagers et 8 membres d'équipage est abordé par des pirates armés qui détruisent ses équipements de communication et le détournent vers les eaux chinoises. Les assaillants s'emparent de 1,6 millions de dollars US, à bord pour le compte d'une compagnie de transports de fonds, ainsi que des objets de valeur dont disposent les passagers. Finalement arrêtés en Chine, leur chef sera condamné à mort tandis que ses comparses purgeront de lourdes peines de prison. Toujours en juin, le Hye Mieko est attaqué, alors qu'il se trouve dans les eaux territoriales cambodgiennes, par une embarcation qui, selon le propriétaire du navire, serait similaire aux embarcations utilisées par les douanes chinoises. Les pirates détournent le navire vers Shanwei dans la province de Guangdong. Une fois à destination, le navire est arraisonné pour contrebande par les autorités locales qui saisissent la cargaison composée de cigarettes et d'appareils de prises de vues d'une valeur de 2 millions de dollars

8. International Maritime Bureau, Piracy Report 1995 ; New Straits Times, 2.3.95. 
Singapour. En juillet, alors qu'il navigue sur le fleuve Musi près de Palembang, le Trans Hercules est attaqué par six pirates munis d'armes blanches qui s'enfuient avec 1700 dollars US et des biens personnels de l'équipage. En août, quatre pirates indonésiens s'emparent d'un bateau de pêche malaisien au large de Kuala Selangor dans le Détroit de Malacca et par radio réclament une rançon de 30000 ringgit au propriétaire du bateau. Celui-ci parvient toutefois à prévenir la police maritime qui, après un échange de coups de feu, coule l'embarcation des pirates, âgés de 25 à 41 ans, avant de les arrêter (9). Le 13 septembre 1995, le Anna Sierra, transportant 12 000 tonnes de sucre pour une valeur de 5 millions de dollars US à destination de Manille, est intercepté dans le Golfe de Siam par 30 pirates masqués et armés de mitraillettes, pistolets et couteaux. L'équipage, composé de 23 membres, est neutralisé pendant deux jours tandis que les pirates repeignent une partie du navire. Le 15 septembre, huit hommes d'équipage sont mis à la mer sur un radeau de fortune à environ $180 \mathrm{~km}$ des côtes du Viêt Nam. Les pirates tirent sur l'embarcation sans toutefois faire de blessé. Les 14 membres d'équipage restants sont mis à la mer quelques heures plus tard dans un canot de sauvetage. Les pirates changent le nom du navire qui devient Artic Sea, enregistré au Honduras, et font route vers le port de Beihai dans la région autonome chinoise de Guangxi, où ils arrivent une semaine après l'attaque. Les documents falsifiés indiquent que la cargaison, d'origine thaïe, a été chargée au Brésil. L'enquête montrera qu'elle avait été vendue illégalement à un marchand de Beihai début septembre, c'est-à-dire avant l'attaque, pour un prix bien inférieur au prix du marché. Le bateau est détenu par les autorités de défense frontalière chinoise. L'examen des passeports de l'équipage indique que douze d'entre eux ont un passeport indonésien et deux un passeport malaisien. Il montre également que s'ils sont arrivés par avion à Bangkok début août en provenance de Singapour, ils ont quitté la Thaïlande sans passer par les services d'immigration. Cinq mois plus tard, les autorités chinoises (Bureau de sécurité publique) invitent le propriétaire à récupérer son navire moyennant le paiement d'une somme de 400000 dollars US, censés représenter les frais qu'elles ont supportés depuis l'arrivée du navire. Le propriétaire refuse. Les pirates sont rapatriés entre fin décembre 1996 et janvier 1997 sans apparemment avoir été interrogés par les autorités chinoises. Ces dernières vendront finalement la cargaison de sucre aux enchères en août 1997 sans avoir consulté les propriétaires (10). En

9. International Maritime Bureau, Piracy Report 1995; New Straits Times, 9.8.95.

10. International Maritime Bureau, Piracy Report 1995; New Straits Times, 12.3.96; Piracy and Armed Rohbery Against Ships, A special report, 1998: 40-45. 
octobre 1995, six pirates tirent sur un bateau de pêche au large de Kabasalan dans l'île de Mindanao et abattent deux pêcheurs. Le reste de l'équipage se jette à la mer et les assaillants s'emparent du bateau. En novembre cinq pirates puissamment armés tirent sur un navire au large de Pulau Ligitan (Sabah). Après avoir abordé, les assaillants s'emparent des espèces et du système de positionnement global du navire avant de s'enfuir vers les eaux territoriales philippines. En décembre, le Xiamen Bridge est abordé au large de Hong Kong par des hommes armés en uniformes militaires chinois. Les assaillants donnent l'ordre de faire route vers Dangan Liedao (Chine). Après avoir ouvert sept containers, les pirates s'aperçoivent qu'ils ne peuvent s'emparer de la cargaison et quittent le navire. L'attaque aura duré près de sept heures.

\section{Année 1996(11)}

En février, cinq pirates abattent neuf pêcheurs au large de l'île de Basilan (Philippines). Le même mois, après un échange de tirs avec des bateaux chinois au large du port de Subic ( $80 \mathrm{~km}$ au nord-ouest de Manille), la marine de guerre philippine arrête 20 pirates à bord d'un bâtiment dont la cabine de pilotage est blindée et la proue renforcée pour éperonner. En plus de cartes marines provenant de l'armée chinoise, le bateau possède deux enregistrements administratifs effectués dans des localités différentes de la province de Guangdong. Des officiels philippins affirment à cette occasion que certains éléments de la marine de guerre chinoise opérant à partir de bases à Shantou et Shanwei (Guangdong) sont engagés dans la piraterie et la contrebande en Mer de Chine(12). Toujours en février, Semporna, une petite ville sur la côte est de Sabah, est attaquée par deux fois par des pirates, attaques qui causent plusieurs milliers de ringgit de dégâts(13). En mars, le cargo Elaine en route pour Sihanoukville (Cambodge) disparaît en Mer de Chine méridionale avec une cargaison de cigarettes d'une valeur de 3 millions de dollars US. En mai, un petit bateau de pêche est attaqué de nuit par sept pirates au large de Perak dans le Détroit de Malaka. Ils s'emparent d'un radar, d'espèces et du produit de la pêche (14). Le même mois, les pirates responsables de l'attaque du Anna Sierra en 1995, détournent le Samudra Samrat au large de Singapour, changent son nom en Celtic Ranger et tentent de décharger illégalement sa cargaison à Fengcheng (Chine). Pourtant alertées par le Bureau maritime inter-

11. International Maritime Bureau, Piracy Report 1996 (sauf autre indication).

12. New Straits Times, 23.2.96.

13. Berita Harian, 27.03.96.

14. Harian Watan, 25.05.96. 
national, les autorités locales refusent d'intervenir et le navire repart le lendemain laissant tous ses documents et les passeports de l'équipage (23 Indonésiens). Le bateau et sa cargaison, d'une valeur de 2,5 millions de dollars US, destinée au Viêt Nam, ne seront apparemment jamais retrouvés (15). En novembre, un tanker malaisien, le Suci, transportant plus de 2600 tonnes de fuel pour une valeur de 1,5 millions de ringgit à destination de Sandakan (Sabah) est intercepté par six pirates à cinquante minutes du phare de Horsburgh, près de Singapour. Les assaillants forcent l'équipage à conduire le navire vers le phare au voisinage duquel quatre autres pirates montent à bord. Le lendemain, tout l'équipage excepté deux mécaniciens, est mis sur un canot de sauvetage. Entre-temps une partie du bateau a été repeint, son nom a été changé en Glory II, ainsi que son lieu d'enregistrement qui devient San Lorenzo au Honduras (16). Les deux mécaniciens sont retrouvés au large d'Ujung Pandang (Sulawesi) près d'un mois plus tard. Le navire n'a apparemment jamais été retrouvé. Toujours en novembre, six pirates interceptent un cargo maltais au large de Mindanao, neutralisent l'équipage et s'échappent avec montres, jumelles, bijoux et espèces d'une valeur avoisinant 17000 dollars US (17). A la même époque le même type d'attaque produisant un butin similaire est perpétré à deux reprises dans le Détroit de Gelasa (Indonésie). En décembre, six pirates interceptent un navire au large de Hong Kong et s'emparent de cinq containers de produits alimentaires congelés, d'une valeur de 2,5 millions de dollars Hong Kong, qu'ils transfèrent sur un autre navire.

Année 1997(18)

En février, sept pirates suspectés d'appartenance à la guérilla islamique, détournent un ferry-boat au large de Sirawai (Philippines), dévalisent les 50 passagers avant d'en abattre trois. Une attaque similaire se produit dans l'archipel de Basilan, au cours de laquelle deux passagers sont abattus (19). En février, le tanker Lung Shun No. 8 est contraint de stopper dans le Golfe de Thaïlande après avoir essuyé des rafales de mitraillettes provenant de bateaux de pêche. Il est alors escorté vers l'île de Ko Rong puis le lendemain vers l'île de Ronde. Suite à l'arrivée de trois bateaux de la marine de guerre

15. Piracy and Armed Robbery Against Ships, A special report, 1998 : 45-46.

16. Mingguan Malaysia, 24.11.96; Berita Minggu, 24.11.96; Harian Watan, 25.11.96.

17. New Sunday Times, 4.5.97.

18. International Maritime Bureau, Piracy Report 1997 (sauf autre indication).

19. International Maritime Burcau, Piracy Report 1997; Berita IIarian, 9.5.97. 
cambodgienne, le tanker peut reprendre sa route non sans que les effets personnels de l'équipage, les équipements et la cargaison aient été subtilisés. En mars 1997, un cargo en provenance de Singapour à destination de la Corée du Sud est intercepté au large de l'archipel de Natuna par une dizaine de pirates armés de revolvers et fusils M-16. Les pirates, supposés thaïs, neutralisent l'équipage et vont rester maîtres du navire pendant six jours durant lesquels ils font transférer sur un autre navire, par l'équipage lui-même, 13 containers de cigarettes d'une valeur de plus de 8 millions de ringgit. Ils s'emparent également de tous les documents relatifs au bateau, des cartes, des passeports et de l'équipement radio(20). Toujours en mars, alors qu'il jette l'ancre au large de Laem Chabang (Thaïlande), quinze jeunes femmes et cinq hommes se glissent à bord d'un porte-container. Rapidement découvertes par le capitaine, les jeunes femmes expliquent qu'elles ont payé pour leur transport jusqu'au navire ainsi que pour leur escorte masculine mais qu'elles n'ont pas eu le temps de faire valoir leurs charmes auprès de l'équipage contre rémunération. L'une d'elle prévient même que le navire sera attaqué la nuit-même. À la nuit tombée, deux embarcations s'approchent en effet du navire et les pirates tentent de grimper par la chaîne de l'ancre mais l'équipage, sur ses gardes, parvient à les mettre en fuite. En juillet, cinq pirates abordent un yacht dans la baie de Jakarta, blessent grièvement un membre d'équipage et s'enfuient avec 12000 dollars US et des équipements. En octobre, le Vosa Carrier, parti de Hong Kong pour Hai Phong est intercepté au large de l'île de Waglan par une vedette rapide chinoise avec à son bord douze hommes en tenuc militairc, armés de mitraillettes. Alors que le navire est détourné vers Huilai (Guangdong), les cabines sont mises à sac par les pirates qui s'emparent des espèces et l'équipage est contraint de signer un document dans lequel il reconnaît se livrer à des opérations de contrebande. À Huilai, les autorités locales, s'appuyant sur ces déclarations, accusent le capitaine d'activités illicites. Le navire est alors conduit au port voisin de Paotai où la police décharge et confisque tous les containers, représentant une valeur de 20 millions de dollars Hong Kong. Le navire et l'équipage sont finalement relâchés un mois plus tard, non sans le versement préalable d'une amende de 100000 dollars US en espèces à la police. Toujours en octobre, six pirates à l'accent indonésien portant des uniformes de la police maritime, armés de mitraillettes et revolvers, interceptent un bateau de pêche au large de Pulau Ketam dans le Détroit de Malacca. Non satisfaits de

20. New Straits Times, 12.3.97; Utusan Malaysia, 13.3.97. 
la petite somme d'argent en possession des deux pêcheurs, les pirates les obligent à monter sur leur bateau et se dirigent vers un autre navire de pêche dans la même zone. Déçus là encore par la maigreur du butin en possession des deux pêcheurs, ils emmènent finalement les quatre pêcheurs chinois dans un endroit isolé du port de Dumai à Sumatra. Là, un Chinois leur traduit les exigences des pirates pour leur libération, à savoir une somme de 20000 ringgit par personne. Après négociation, les pêcheurs acceptent de payer 8000 ringgit chacun si les pirates les laissent rentrer chez eux pour réunir l'argent. En attendant, l'interprète chinois est retenu en otage jusqu'à ce que les pêcheurs aient transféré la somme demandée par voie télégraphique (21). En novembre, le tanker Rahah est intercepté entre Singapour et Brunei par dix pirates munis d'armes à feu. Les cabines sont mises à sac, les pirates s'emparant d'espèces et objets de valeur, des certificats du navire, de documents, passeports, carburant et provisions. Ils quittent le navire trois jours plus tard. Le même mois le Theresa, un tanker en provenance de Belawan (Sumatra) et faisant route vers Huizhou (Chine), est intercepté en Mer de Chine méridionale par une trentaine de pirates armés de fusils M-16. Après avoir blessé un membre de l'équipage, ils ordonnent le transfert du carburant et de l'eau potable sur leur bateau, puis s'emparent des espèces, de provisions et d'équipements. Ils quittent le tanker quatre heures après l'attaque. Toujours en novembre, le tanker Atlanta 95 est attaqué dans le Détroit de Riau (Sumatra). Le bateau est détourné vers le Golfe de Thaïlande où la cargaison de fuel est transférée sur un autre tanker. Les pirates s'emparent également d'équipements et de documents. Ils abandonnent le navire trois semaines après l'attaque.

\section{Janvier-mai 1998(22)}

En janvier 1998, le Tioman 1, un tanker faisant route vers le Golfe de Thaïlande avec une cargaison de trois millions de litres de fuel, est intercepté par dix pirates au large de Pulau Aur (Johor, Malaisie). Après avoir neutralisé l'équipage, ils s'emparent de tout l'équipement de communications, des espèces et siphonnent un million de litres de fuel dans un autre tanker(23). L'attaque aura duré quinze heures. D'après les témoignages de l'équipage, les pirates s'exprimaient en malais, thaï, chinois, birman et anglais.

21. The Star, 13.11.97.

22. International Maritime Bureau, Piracy Report 1.01-31.03.98 (sauf autre indication).

23. International Maritime Bureau, Piracy Repurt 1.01-31.03.98; New Straits Times, 12.5.98. 
Le 17 avril un tanker malaisien venant de Singapour et à destination de Hô Chi Min Ville est intercepté de nuit par douze pirates armés uniquement de machettes, au large de l'île de Tioman en Mer de Chine méridionale. Avec un équipage de 21 marins, il transporte plus de 9500 tonnes de gaz et près de 1600 tonnes de pétrole, le tout d'une valeur de 1,5 millions de dollars US. Les douze pirates, probablement indonésiens en majorité, détruisent une partie des équipements de communications, repeignent le navire, changent son nom et lieu d'enregistrement (Honduras) puis commencent à siphonner la cargaison sur un navire battant pavillon chinois. Les deux bâtiments sont arraisonnés par la police maritime chinoise le 26 avril alors que 6000 tonnes ont déjà été transférées. Le tanker, son équipage ainsi que les 12 pirates indonésiens sont alors retenus par les autorités chinoises dans le port de Haikou (île de Hainan) le temps de l'enquête (24). Le navire et son équipage sont finalement relâchés fin mai (25). Entre avril et mai, cinq attaques sur des bateaux de pêche malaisiens se produisent dans le Détroit de Malacca, au large de Muar (Johor, Malaisie). Les pirates, armés de fusils M-16, prennent en otage l'équipage et envoient l'un des pêcheurs à terre chercher la rançon demandée (entre 2000 et 5000 ringgit par personne)(26).

\section{Statistiques et cartographie}

Il convient tout d'abord de signaler la précision relative des statistiques fournies. Le Bureau maritime international estime en effet que la moitié des cas seulement lui sont rapportés. Plusieurs raisons sont avancées pour expliquer cette distorsion. La première est technique avec les difficultés de communications entre les navires et les autorités à terre. La seconde est économique avec d'une part la crainte qu'une enquête ne perturbe la mission du navire en provoquant une immobilisation plus ou moins prolongée pour interroger l'équipage, d'autre part l'éventualité d'une augmentation des primes d'assurances pour les propriétaires de navires. Par ailleurs, si le vol concerne uniquement des biens personnels de l'équipage, le capitaine sera fortement tenté de ne pas rapporter l'affaire aux autorités car la perte de temps occasionnée entraînerait une hausse du coût du transport sans commune mesure avec le coût des biens dérobés (27). La troisième raison est poli-

24. The Star, 2.5.98; Berita Harian, 5.5.98; Utusan Malaysia, 5.5.98; New Straits Times, 19.05.98; The Star, 10.06.98.

25. Berita Harian, 4.06.98; Berita Harian, 14.06.98.

26. New Sunday Times, 31.05.98; The Star, 1.06.98.

27. Pirary and Armed Robhery Against Ships, A special report, $1998: 4$. 


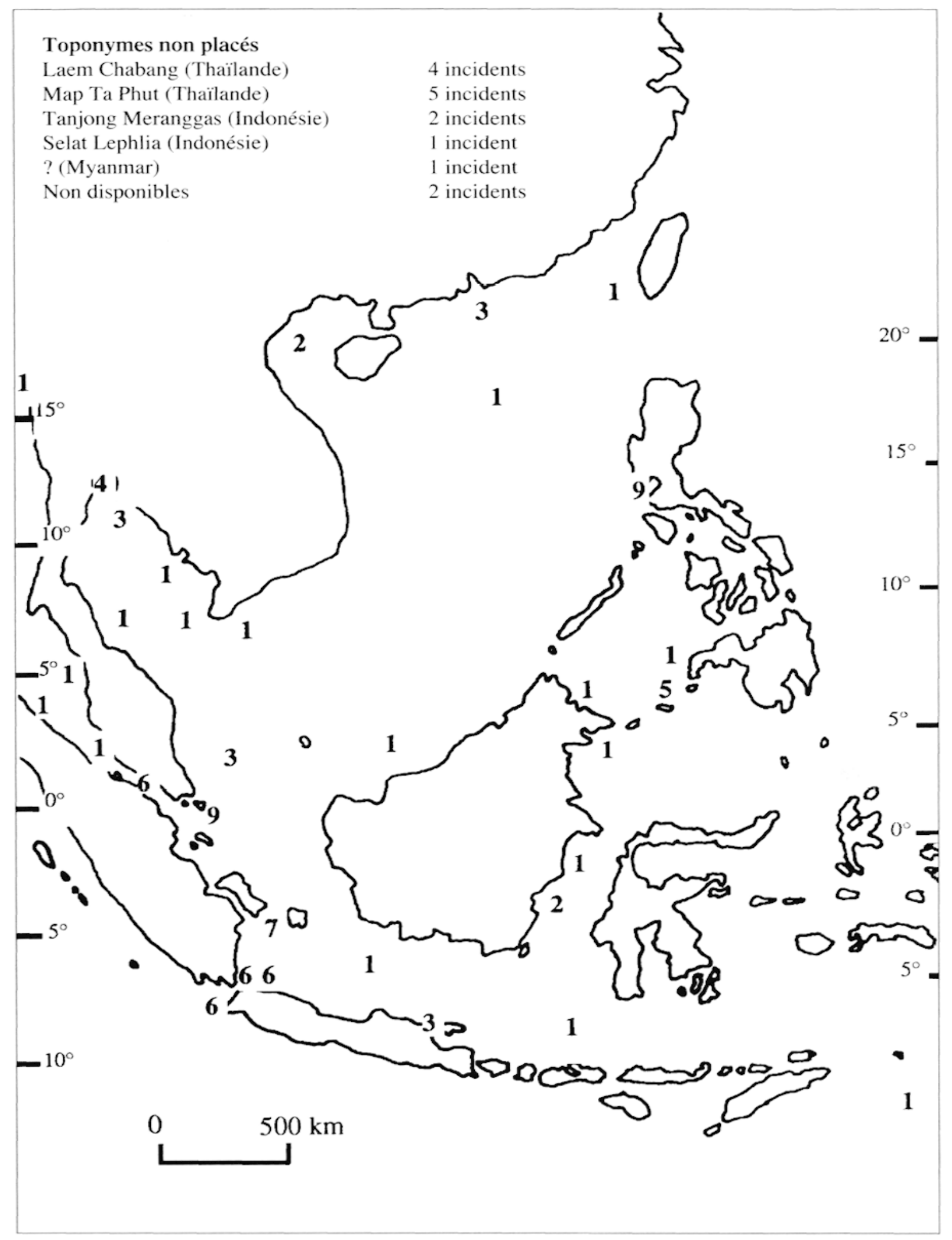

Localisation des incidents en Méditerranée Sud-Est asiatique rapportés au Bureau maritime international pour l'année 1997 
tique avec la crainte que le rapport n'embarrasse le pays dans les eaux duquel s'est déroulée l'attaque. D'autres raisons invoquées sont la crainte que la diffusion d'informations sur le succès d'un acte de piraterie ne nuise à la réputation du capitaine ou que soit mise en doute l'intégrité des autorités à qui l'incident devrait être rapporté(28). On notera par ailleurs la discordance entre les statistiques fournies par le Regional Piracy Centre et la police maritime malaisienne qui tient au fait que les incidents impliquant des bateaux de pêche sont rarement rapportés au Bureau maritime international.

Comme au début du XIX ${ }^{\mathrm{e}}$ siècle, certaines zones de la Méditerranée SudEst asiatique sont plus particulièrement propices aux attaques de pirates. Les rapports du Regional Piracy Centre montrent ainsi la prédominance de l'Indonésie dans les statistiques.

Avant 1989, le Détroit de Malacca, emprunté quotidiennement par environ 2500 navires et embarcations de tous types, était considéré comme relativement sûr avec une moyenne de sept incidents rapportés annuellement (29). Cette sûreté prévalait également à la même époque dans une zone beaucoup plus vaste comprenant le Détroit de Malacca, le Détroit de Singapour et la Mer de Chine méridionale (en gros la partie maritime de la province actuelle de Riau), qui n'a pas connu plus d'une douzaine d'attaques par an entre 1981 et 1988, sauf en 1982-83(30). Entre le début 1990 et la mi-1992, l'archipel de Riau devient la zone à plus haut risque au niveau mondial avec au moins 200 attaques perpétrées. En 1992, $47 \%$ des attaques enregistrées en Asie du SudEst sont concentrées dans le voisinage de l'île de Bintan et du Phillip Channel (31). Les attaques diminuent très sensiblement à partir de la mi1992(32). Cette recrudescence subite des attaques a été attribuée par certains au développement rapide de la région appelée «triangle de croissance» Singapour-Johor-Riau. Ce développement rapide, accompagné d'immigration massive, aurait engendré des déséquilibres économiques dont la piraterie serait une des conséquences (33).

Après 1992, les foyers d'attaques se déplacent en Mer de Chine et dans le reste de l'Indonésie. Ainsi en 1993, près des trois quarts des incidents surve-

28. Jon Vagg, «Rough Seas? Contemporary Piracy in South East Asia», British Journal of Criminology, 35(1), $1995: 65$.

29. The Star, 4.5.98.

30. Jon Vagg, 1995 : 69.

31. International Maritime Bureau, Piracy Report 1992.

32. Jon Vagg, $1995: 63,70$.

33. Jon Vagg, $1995: 75$. 
nus en Méditerranée Sud-Est asiatique ont lieu en Mer de Chine. En 1995, Indonésie et Mer de Chine représentent respectivement $28 \%$ et $36 \%$ du nombre d'incidents. En 1997, près de $44 \%$ des incidents se produisent en Indonésie en dehors du Détroit de Malacca. Cette recrudescence en Mer de Chine pourrait s'expliquer en partie par les fortes taxes à l'importation prélevées par les autorités chinoises, une situation qui encourage la contrebande (34). Les deux zones sont par ailleurs très vastes et comportent de nombreuses îles inhabitées qui peuvent servir de refuge et de base aux pirates. La surveillance et le contrôle sont par conséquent rendus difficiles.

Les statistiques de la police maritime malaisienne font apparaître un autre foyer de piraterie, Sabah, qui concentre plus de $87 \%$ des incidents recensés par cette autorité en Malaisie entre 1991 et 1996. En novembre 1995, le ministère de l'intérieur malaisien annonçait ainsi que la police avait recensé 48 bandes de pirates dans les eaux territoriales malaisiennes, dont 11 actives à Sabah (35). Les principales zones concernées sont Semporna, Kunak, Lahad Datu, Felda Sahabat et Tawau. La fréquence des actes de piraterie dans cette zone est d'abord attribuée au facteur géographique. Ainsi, la seule côte orientale est longue de plus de $1400 \mathrm{~km}$ et comporte plus de 500 îles. La proximité des eaux internationales joue aussi un rôle important. Les plus proches ne sont qu'à une vingtaine de minutes(36) et Semporna, Sandakan, Lahad Datu n'en sont éloignés que de 2 à 4 heures pour des bateaux rapides. Par ailleurs, la police maritime malaisienne attribue les problèmes de piraterie dans cette zone essentiellement à des étrangers ou des semi-locaux résidant à Sabah, des immigrants philippins en particulier(37). A Sabah, les victimes sont surtout les pêcheurs, les marchands faisant du troc et plus rarement les gros bateaux marchands. Parfois armés, les pirates opéreraient de manière indépendante pour voler bateaux, moteurs, générateurs, téléphones cellulaires, montres, bijoux en or, fuel, espèces et prises faites par les pêcheurs.

34. Utusan Malaysia, 5.5.98.

35. Berita Harian, 27.03.96.

36. Utusan Malaysia, 8.9.97.

37. New Straits Times, 6.7.97. 
Nombre d'incidents rapportés (Bureau maritime international)

\begin{tabular}{|c|c|c|c|c|c|c|c|c|}
\hline & 1991 & 1992 & 1993 & 1994 & 1995 & 1996 & 1997 & $1998^{(1)}$ \\
\hline Indonésie & 55 & 49 & 10 & 22 & 33 & 57 & 47 & 8 \\
\hline Malaisie & 1 & 2 & & 4 & 5 & 5 & 4 & 2 \\
\hline Dét. Malacca & 32 & 7 & 5 & 3 & 2 & 3 & & \\
\hline Singapour & & & & 3 & 2 & 2 & 5 & \\
\hline Philippines & & 5 & & 5 & 24 & 39 & 15 & 6 \\
\hline Thaïlande & & & & & 4 & 16 & 17 & \\
\hline Viêt Nam & & & & 2 & 4 & & 4 & \\
\hline Cambodge & & & 1 & 1 & 1 & 1 & 1 & \\
\hline Birmanie & & & & & & 1 & 2 & \\
\hline $\begin{array}{l}\text { M. de Chine } \\
\text { mérid. }\end{array}$ & 14 & 6 & 11 & 6 & 3 & 2 & 6 & \\
\hline Golfe du Tonkin & & & 1 & & & & & \\
\hline HK-L-H(2) & & & 33 & 12 & 7 & 4 & 1 & \\
\hline C-HK-M (3) & & & 1 & 4 & 31 & 9 & 5 & \\
\hline Dét. Taiwan & & & 1 & & 2 & & & \\
\hline Tot. Méd. SEA & 102 & 69 & 62 & 63 & 118 & 139 & 107 & 16 \\
\hline Tot. mondial & 107 & 106 & 103 & 90 & 188 & 228 & 229 & 26 \\
\hline$\%$ & 95 & 65 & 60 & 70 & 63 & 61 & 47 & 61 \\
\hline \multicolumn{9}{|c|}{$\begin{array}{l}\text { (1) de janvier à mars } \\
\text { (2) Hong Kong - Luzon - Hainan } \\
\text { (3) Chine - Hong Kong - Macao }\end{array}$} \\
\hline
\end{tabular}

Attaques à main armée dans les eaux malaisiennes, 1991-1996 (bateaux malaisiens)

(Source : police maritime malaisienne in New Straits Times, 6/7/97)

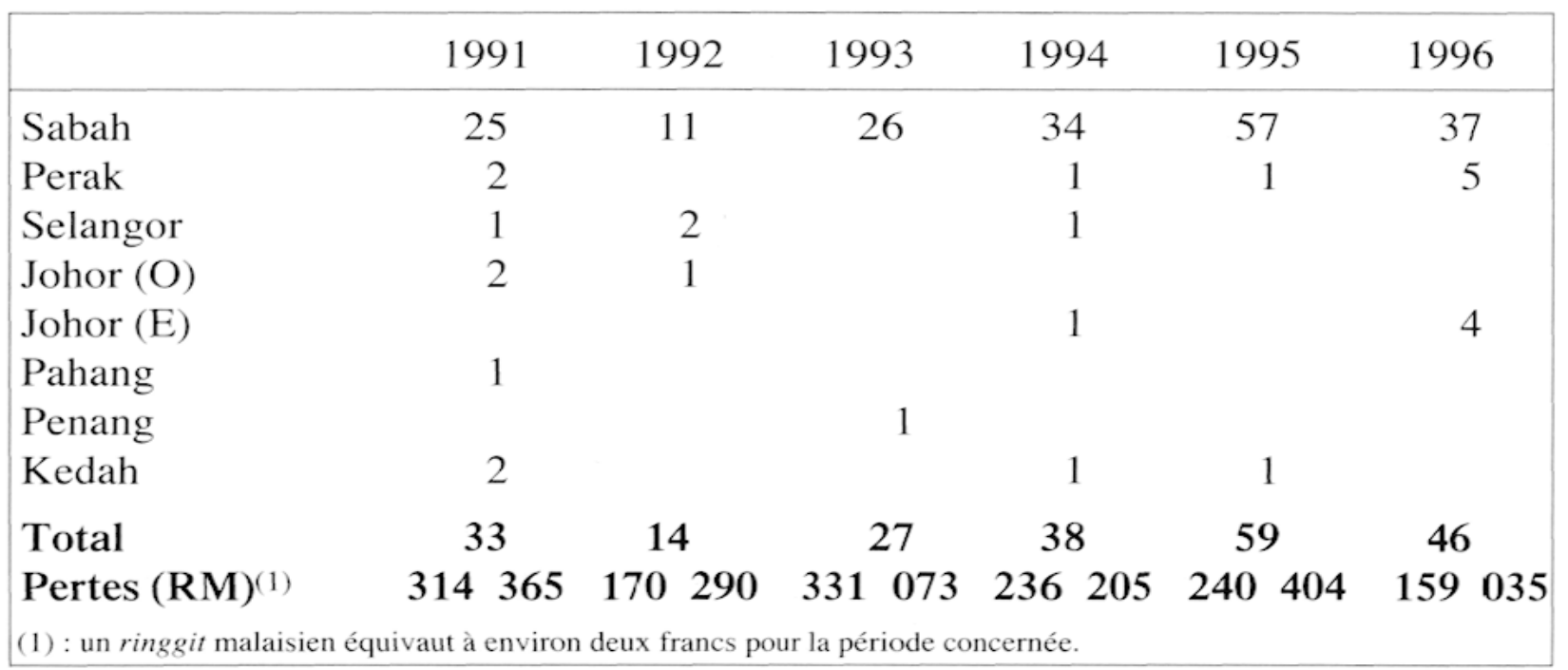


Au début du XIX ${ }^{\mathrm{e}}$ siècle, les deux grands foyers de piraterie étaient l'archipel de Riau-Lingga (avec plus de 150 navires)(38) et la côte ouest de Mindanao (39). Près d'un siècle et demi plus tard, l'archipel de Riau-Lingga n'est plus qu'un foyer épisodique (1990-1992) et le foyer de Mindanao semble s'être déplacé vers la côte est de Sabah. Toutefois, pour les années 1995-96 on ne dispose pas de toutes les localisations des incidents aux Philippines qui ont vu une recrudescence subite de la piraterie. Par ailleurs, si les chiffres de 1997 montrent bien que la piraterie est toujours présente en Mer de Sulu en général et sur la côte ouest de Mindanao en particulier, la région la plus touchée paraît être celle de Manille. Rappelons que ces chiffres concernent presque uniquement les gros bateaux marchands et par conséquent seul l'examen des statistiques de la police maritime philippine permettrait d'avoir une idée plus exacte de la situation dans cette zone. D'une manière générale, les statistiques des polices maritimes des autres États de la Méditerranée Sud-Est asiatique montreraient probablement l'existence d'autres foyers comparables à celui de la côte orientale de Sabah, foyers qui ne sont pas perceptibles au travers des statistiques du Bureau maritime international. Les témoignages et les statistiques montrent que parallèlement à ces foyers bien délimités existe aujourd'hui une zone redoutée beaucoup plus vaste, la Mer de Chine, où sévit un autre type de piraterie.

\section{Stratégies et réseaux}

Les cas décrits plus haut montrent clairement qu'il existe aujourd'hui deux types de piraterie. D'une part une piraterie très localisée que l'on pourrait qualifier «d'artisanale», qui s'attaque notamment aux bateaux de pêche ou s'empare sur les gros navires marchands des objets de valeur de l'équipage et de quelques équipements, d'autre part une piraterie qui, s'appuyant sur des réseaux internationaux (on voit mal une bande de pirates isolée disposer d'un tanker pour siphonner un million de litres de produits pétroliers), évolue avec de grands moyens à l'échelle de la Méditerranée Sud-Est asiatique et se livre à la contrebande des cargaisons volées ou même à des opérations de transport clandestin sur des navires «fantômes». L'accroissement du nombre d'attaques relevant de ce type d'organisation sophistiquée suggère une augmentation du nombre des gangs criminels à qui de telles attaques peuvent rapporter des profits considérables.

39. Ibid. : 235. 
Types d'incidents rapportés (Bureau maritime international)

\begin{tabular}{|lrrrr|}
\hline & 1994 & 1995 & 1996 & 1997 \\
\hline détournement & 4 & 5 & 2 & 7 \\
abordage & 35 & 53 & 69 & 76 \\
détention & 4 & 5 & 1 & 3 \\
coups de feu & 1 & 3 & 8 \\
tentative & 16 & 22 & 22 & 11 \\
vol & 3 & 1 & 1 & 2 \\
info. non disp. & 1 & 31 & 41 & \\
Total & 63 & $\mathbf{1 1 8}$ & $\mathbf{1 3 9}$ & $\mathbf{1 0 7}$ \\
\hline
\end{tabular}

Types de navires attaqués (Bureau maritime international)

\begin{tabular}{|lrrrrr|}
\hline & $\mathbf{1 9 9 3}$ & $\mathbf{1 9 9 4}$ & $\mathbf{1 9 9 5}$ & $\mathbf{1 9 9 6}$ & $\mathbf{1 9 9 7}$ \\
\hline cargo polyvalent & 20 & 17 & 13 & 19 & 17 \\
porte-container & 10 & 11 & 6 & 9 & 16 \\
transp. en vrac & 12 & 12 & 21 & 28 & 24 \\
tanker & 8 & 9 & 14 & 14 & 17 \\
transp. LPG/LNG & 1 & 4 & 4 & 6 & 6 \\
tanker prod. chim. & & 2 & 2 & 7 & 2 \\
transp. véhicules & 1 & 2 & & 2 & \\
barge & 1 & & & 1 & \\
nav. entrepôt & 1 & & & & 1 \\
transp. grumes & 1 & & 3 & & \\
chalutier & 1 & 1 & 1 & 5 & \\
ferry/jetfoil & 1 & 1 & 2 & 1 & 3 \\
bat. de pêche & 1 & & & & 3 \\
yacht & 1 & 4 & 3 & 5 & 1 \\
autres & 3 & 1 & 49 & 46 & 12 \\
info. non disp & 62 & 63 & $\mathbf{1 1 8}$ & 139 & $\mathbf{1 0 7}$ \\
Total & & & & & \\
\hline
\end{tabular}


D'un certain point de vue, les pirates tirent avantage de la modernisation des transports maritimes. En effet, à l'heure actuelle les navires marchands fonctionnent avec un équipage réduit ( 15 à 20 personnes en moyenne sur les gros bâtiments). Par ailleurs, la réglementation maritime internationale dissuade par exemple les tankers de s'équiper d'armes à feu pour des raisons évidentes de risque d'explosion du navire. Enfin, d'un point de vue technique, les gros navires chargés constituent une proie facile. Ainsi le pont d'un tanker à pleine charge n'est qu'à deux mètres environ au-dessus de l'eau.

En Asie du Sud-Est, la plupart des attaques ont lieu de nuit, entre $22 \mathrm{~h}$ et 4h, lorsque les bateaux sont ancrés ou à quai, avec une prédilection pour les navires de faible tonnage. Les pirates, en général entre cinq et dix individus, sont équipés de petits bateaux rapides; ils montent à bord à l'aide de grappins et de cordes puis menacent l'équipage. Si dans les ports de Chine et de Hong Kong, les pirates cherchent à voler des équipements sans se faire remarquer par l'équipage, à Macau, ils préfèrent investir les ferry-boats et soustraire les objets de valeur des passagers et de l'équipage. Ailleurs, ils se font parfois passer pour des pêcheurs ou revêtent des uniformes de police. Sur les gros navires marchands, les pirates s'emparent généralement du contenu du coffre-fort, voire du coffre-fort lui-même, ainsi que des espèces et effets de l'équipage. Ce genre d'attaque dure 30 minutes en moyenne et, pour la région considérée, la valeur moyenne du vol est de 5000 dollars US.

Par ailleurs les pirates n'hésitent pas à débarquer, notamment à Sabah (40), pour détrousser les commerçants. Ainsi, en septembre 1985, la petite ville de Lahad Datu sur la côte est de Sabah fut attaquée par une cinquantaine de pirates qui prirent le contrôle de la ville pendant environ une heure. L'attaque fit dix victimes. Ils débarquèrent à la mi-journée en tenue kaki, armés de fusils automatiques M-16, lance-roquettes et grenades. Les habitants n'y prêtèrent pas vraiment attention croyant qu'il s'agissait de membres de la police. Tandis qu'une fusillade se déclenchait au marché aux poissons et au port, plusieurs pirates prirent position aux quatre coins de la ville pour empêcher l'arrivée de renforts de police. Après avoir dévalisé une banque et une bijouterie, les pirates se retirèrent en entendant l'appel à la prière d'une mosquée voisine. Le lendemain, quatre d'entre eux furent abattus par la police dans une île proche. Bien connue aux Philippines, cette bande est dirigée par un

40. Les débarquements de pirates à Semporna sur la côte est de Sabah ne constituent pas un fait nouveau. Cette petite ville a en effet été attaquée en 1952 et même brièvement contrôlée et pillée en 1954 (K.G. Tregonning, North Borneo, London: HMSO, cité dans Clifford Sather. The Bajau Laut. Kuala Lumpur : Oxford University Press, $1997: 64$ ). 
certain Sarip Ibah (41). Cet épisode n'est pas sans rappeler les attaques commises sur la route de la côte nord de Java au début du XIX ${ }^{\mathrm{e}}$ siècle pour détrousser les véhicules (42).

Toujours sur le plan stratégique, rapports et statistiques convergent pour indiquer l'accroissement des attaques violentes, en particulier des assassinats. Les descriptions données au début de cet article montrent que les attaques à la mitraillette ne sont pas rares, les pirates usant même parfois de lance-roquettes et d'explosifs. Entre 1980 et 1984, 2400 réfugiés du Viêt Nam ont été jetés par dessus bord par des pirates en Mer de Chine méridionale. Entre 1980 et 1985, au moins 1375 personnes ont été assassinées au large de la Thaïlande(43). Dans la seule Malaisie, entre 1990 et septembre 1997, neuf pêcheurs ont été abattus par les pirates et 29 ont été blessés (44). Au niveau mondial en 1997, 51 marins ont été tués et plus de 400 membres d'équipage pris en otage, deux chiffres qui traduisent une augmentation de plus de $100 \%$ par rapport à 1995 ou 1996. En Méditerranée Sud-Est asiatique uniquement, 7 marins ont été abattus et 171 membres d'équipage pris en otage au cours de cette même année 1997.

Contrairement au début du siècle dernier, pour lequel on dispose de données sur la responsabilité des attaques, les informations semblent aujourd'hui très fragmentaires ou, à tout le moins, ne sont pas publiées et, qui plus est, nombreuses sont les attaques non rapportées. Une chose est certaine, les pirates qui détournent les gros cargos et les tankers pour s'emparer de leur cargaison font partie de réseaux internationaux qui retirent probablement les plus gros bénéfices. Le cas de la cargaison de sucre d'une valeur de 5 millions de dollars US, vendue par les pirates au quart de sa valeur dès avant l'attaque, en est un bon exemple. Les grandes opérations de piraterie sont minutieusement préparées avec accès aux informations sur le trafic maritime et sur le navire visé pour connaître le détail de sa cargaison et pouvoir falsifier ses documents d'identification. Si la cargaison est composée d'une grande quantité de denrées comme du caoutchouc, du sucre ou de l'huile de palme, elle est évidemment difficile à écouler directement sur le marché. Il faut donc que l'opération soit commanditée par l'acheteur lui-même ou que les pirates aient l'appui d'un réseau d'écoulement. Là encore, l'exemple de la 
cargaison de sucre, vendue dans la zone autonome du Guangxi à un marchand local, est éclairant.

Une méthode encore plus sophistiquée s'est développée en ExtrêmeOrient dès les années 1970 et surtout depuis la fin des années 1980. Il s'agit du «bateau fantôme » ou «kapal hantu». Les Philippines étaient particulièrement réputées dans les années 1980 pour ce genre d'opérations. Dans ce cas, c'est moins la cargaison qui intéresse les pirates (les navires victimes sont d'ailleurs souvent vides) que le bateau lui-même. L'opération suppose que, par un système d'agents et de contacts, le gang organisé apprenant qu'un affréteur cherche un navire pour transporter sa cargaison s'emploie à en voler un. Celui-ci est détourné, l'équipage parfois jeté par dessus bord et la cargaison éventuelle vendue. Aux Philippines dans les années 1980, les navires étaient détournés et livrés en trois jours sur commande pour une somme de 300000 dollars US. A partir de là, le navire devient «navire fantôme », une situation rendue possible grâce au système d'inscriptions provisoires accordées sans discernement par certaines autorités. Les consulats du Panama, du Honduras, de Belize et de St. Vincent semblent particulièrement prisés pour effectuer ce genre d'inscription. Les demandes sont soumises par des «bureaux maritimes», des «assistances maritimes» ou des «compagnies maritimes» basées en Asie du Sud-Est. Le «certificat d'enregistrement provisoire» ou «Patente Provisional de Navegación» est valable pour trois mois. Le coût d'enregistrement d'un navire «fantôme» équivaut à plusieurs fois le coût d'enregistrement standard. Les documents soumis lors de l'enregistrement comportent de fausses informations, concernant notamment les caractéristiques et le propriétaire du navire. Ces falsifications ainsi que son enregistrement sous plusieurs autres noms rendent bien sûr sa localisation très difficile. L'enregistrement du bateau «fantôme» peut même s'effectuer avant qu'il n'ait été détourné. Munis de ces nouveaux papiers, les propriétaires du navire "fantôme» peuvent alors commettre des vols de cargaison. L'enregistrement provisoire leur garantit par ailleurs une protection en cas de poursuites judiciaires. Les services du navire «fantôme» sont proposés à un affréteur pressé d'acheminer une cargaison. Le destinataire officiel attendra en vain la sienne qui est déchargée dans un autre port et vendue à un consignataire parfois complice. L'opération réalisée, le navire est à nouveau enregistré sous une nouvelle identité «fantôme» et se trouve ainsi prêt pour une seconde opération de détournement de cargaison. Ce fut le cas du Isla Luzon, détourné en juin 1989 au large de Illigan (Philippines). Renommé Nigel, le navire «fantôme » se livra à plusieurs opérations «commerciales » entre Taiwan, la Chine, la Corée et le Japon. On comprend la difficulté à retrouver 
ces navires en raison des fausses informations sur les documents et leur très brève «durée de vie»(45). Les membres d'équipage recrutés sur ces navires sont généralement des Thaïs, des Birmans ou des Philippins dont le salaire peut atteindre 300 à 500 dollars US. La première victime de «kapal hantu» en Malaisie a été la société Sime Darby Berhad qui a perdu près de 15 millions de ringgit à cette occasion (46). Les pirates impliqués dans ce type d'attaque sont généralement aussi associés à d'autres activités lucratives : trafic d'armes et de drogues ou rejet illégal de déchets toxiques. Une investigation menée par le Bureau maritime international, à la demande d'une banque asiatique, aurait permis ainsi de découvrir que celle-ci avait été utilisée en 1995 par des pirates, pour des transactions frauduleuses d'un montant global de 60 millions de dollars US (47).

Au début du XIX $\mathrm{X}^{\mathrm{e}}$ siècle, les pirates bénéficiaient de complicités multiples, notamment auprès de la plupart des princes musulmans de l'Archipel qui participaient directement ou indirectement à cette activité, démontrant ainsi l'existence d' «un véritable système économique » (48). Aujourd'hui, selon certaines hypothèses, une part des attaques de piraterie en Méditerranée Sud-Est asiatique seraient soutenues, voire directement conduites, par des agents d'État (49). Ainsi, en Mer de Chine, des équipages ont rapporté à plusieurs reprises avoir été victimes d'attaques de pirates se déplaçant à bord de bateaux pilotés vraisemblablement, selon eux, par des fonctionnaires des douanes chinoises (50). Si ce genre d'attaque a connu une réduction en 1995 et 1996, probablement en partie en raison de l'écho qu'en a donné la presse internationale, l'année 1997 a vu se dérouler trois incidents au cours desquels les navires concernés ont été abordés par des individus armés et portant des uniformes militaires chinois qui ont contraint les navires à se rendre dans un port de Chine méridionale(51). Ces informations, qui reposent plus sur des soupçons que sur des données sûres, sont toutefois très difficiles à vérifier.

45. Piracy and Armed Robbery Against Ships, A special report, 1998 : 32-34.

46. Harian Watan, 28.8.95.

47. Berita Harian, 2.12.1996.

48. Lombard, $1979: 236$.

49. Jon Vagg, $1995: 63$.

50. New Straits Times, 12.03.96.

51. International Maritime Bureau, Piracy Report 1997; Piracy and Armed Robbery Against Ships, A special report, $1998: 9$. 


\section{La lutte contre la piraterie}

Le dossier de la piraterie moderne a été porté pour la première fois à l'attention du Bureau maritime international de la Chambre de commerce internationale en 1983, quand plusieurs gouvernements et organisations internationales ont exprimé leur inquiétude devant l'augmentation des actes de piraterie et brigandage à main armée contre des navires dans certains ports. En novembre 1983, l'International Maritime Organisation (IMO) a adopté la résolution A.545(13) dans laquelle figurent des mesures pour prévenir actes de piraterie et brigandage à main armée contre des navires. Le Marine Safety Committee de l'IMO fait alors de «la piraterie et brigandage à main armée contre des navires » un élément spécifique et permanent de son programme de travail. Devant l'augmentation régulière de ces actes, et réalisant que beaucoup d'attaques ne sont pas signalées à l'IMO, l'assemblée de cette organisation a adopté en 1991 la résolution A.683(17), «Prévention et suppression des actes de piraterie et brigandage à main armée contre des navires», dans laquelle les gouvernements membres sont priés de signaler tous les incidents concernant leurs propres navires à l'organisation et les pays ayant une façade maritime d'accroître leurs efforts pour prévenir et supprimer de tels actes dans leurs eaux. En octobre 1992, le Bureau maritime international ainsi que l'Organisation maritime des Nations Unies ont établi à Kuala Lumpur un Centre de renseignements régional sur la piraterie, financé par les contributions volontaires de compagnies de navigation et de compagnies d'assurances (52). Destiné à recevoir les informations concernant les mouvements suspects de navires, les abordages et les vols à main armée, il collecte essentiellement les données concernant des attaques de bateaux marchands. Ces informations sont ensuite transmises aux navires croisant dans les eaux concernées. La mise en place de ce centre aurait contribué notamment à la diminution du nombre des attaques dans les détroits de Malacca et de Singapour. Par ailleurs, il organise régulièrement des rencontres pour discuter de l'évolution de la situation (53). Cette priorité mise sur la collecte et la centralisation des informations relatives à la piraterie(54) n'est pas sans rappeler l'effort entrepris par les Hollandais dans la première moitié du XIX ${ }^{\mathrm{e}}$ siècle, qui centralisaient à Batavia toutes les informations sur la piraterie dans la région (55).

52. International Maritime Bureau, Piracy Report 1992.

53. A titre d'exemple. le Third International Meeting on Piracy and Phantom Ships suivi par 120 délégués de 22 pays, qui s'est tenu début juin 1998 à Kuala Lumpur (The Star, 8.06.98).

54. Des négociations sont en cours pour rendre obligatoire la déclaration d'actes de piraterie.

55. Lombard, $1979: 243$. 
Par ailleurs, si au début du XIX $\mathrm{X}^{\mathrm{e}}$ siècle, un gros effort fut fait sur le plan de la cartographie(56), aujourd'hui un effort comparable est accompli sur le plan de la détection : à partir des navires eux-mêmes, avec pour les plus récents des systèmes électroniques de sécurité, mais aussi et surtout à partir de puissants radars installés notamment dans le Détroit de Malacca depuis juin 1997 (57).

Le troisième volet de la lutte concerne la coopération internationale pour des missions conjointes de surveillance. Un des facteurs avancés pour expliquer la recrudescence des actes de piraterie est en effet la réduction de la présence des flottes militaires russe et américaine en Asie du Sud-Est depuis une dizaine d'années. Cette réduction n'aurait pas été compensée par une augmentation proportionnelle des moyens de surveillance de la part des pays concernés (58). Les pirates ont pour ainsi dire le champ presque libre et semblent conscients qu'ils courent peu le risque d'être capturés(59). C'est seulement en novembre 1994 que s'est déroulée la première opération de patrouille commune Malaisie-Philippines pour lutter notamment contre les pirates. Lancée à Sandakan (Sabah), elle a mobilisé 500 personnes (60). Parallèlement, le renforcement des patrouilles conjointes Malaisie-Indonésie et un accord conclu entre Singapour et l'Indonésie en 1992 sur des patrouilles communes dans le Détroit de Malacca auraient contribué à réduire considérablement le nombre d'attaques dans cette zone (61).

Le quatrième volet concerne le renforcement des actions de police menées à l'intérieur des eaux territoriales, opérations ponctuelles suite à une attaque ou opérations systématiques notamment pour «purger les îles». Ainsi, à la suite de deux attaques contre la petite ville de Semporna au début de l'année, la Malaisie a créé en mai 1996 une nouvelle unité de police interarmes (terrestre, maritime et aérienne) le Pemerintahan Timur (Commandement Est) chargé de la répression de la piraterie sur la côte est de Sabah (62). Sur le plan des résultats, de 1990 à septembre 1997, la police

56. Ibid. : 244.

57. Harian Watan, 18.10.97; The Star, 23.3.98.

58. Piracy and Armed Robbery Against Ships, A special report, $1998: 5$.

59. Piracy and Armed Robbery Against Ships, A special report, 1998 : 1.

60. New Straits Times, 7.12.95.

61. International Maritime Bureau, Piracy Report 1992; Jon Vaag, 1995 : 77 ; New Sunday Times, 4.5.97; New Straits Times, 5.5.98.

62. Utusan Malaysia, 8.9.97. 
maritime malaisienne a abattu 35 «brigands des mers» et en a arrêté 51 (63), alors que 9 pêcheurs ont été assassinés et 29 autres blessés par les «brigands»(64). Parmi ces victimes figure notamment un pirate surnommé le «Sea King» philippin, Moloi Hijang, alias Moloi Uwah. Ce personnage, célèbre pour avoir mené pendant dix années des attaques au large de la côte est de Sabah, fut abattu par la police maritime en octobre 1996 près de Sandakan (65). Il semble qu'après cette disparition la piraterie ait diminué de manière significative au large de Sabah (66).

\section{Conclusion}

Études et statistiques convergent pour confirmer une recrudescence de la piraterie en Méditerranée Sud-Est asiatique depuis une dizaine d'années. Comme au début du siècle dernier, toute la région n'est pas également touchée. Si à l'époque, la côte sud-est de Sumatra et la côte ouest de Mindanao constituaient les foyers principaux, la situation est aujourd'hui plus complexe car la piraterie existe sous deux formes. La piraterie «artisanale», dont les actions se traduisent le plus souvent par un butin équivalent à quelques milliers de dollars US, opère généralement dans des zones restreintes, par exemple la côte est de Sabah, ou aux abords des grands ports de la région comme Hong Kong, Singapour, Bangkok, Jakarta, Surabaya, Belawan ou Manille. La piraterie "des réseaux internationaux » sévit quant à elle aujourd'hui surtout en Mer de Chine. A la fois marginales mais aussi tentacules du grand banditisme international, ces bandes de pirates généralement cosmopolites, équipées de puissants moyens, s'attaquent aux gros navires marchands dont elles subtilisent la cargaison représentant parfois plusieurs millions de dollars US ou s'emparent du bateau lui-même qui, sous une fausse identité, deviendra "fantôme» et servira à transporter illégalement des cargaisons dans toute la région, voire au-delà. Les mouvements de navires détournés ces dernières années montrent bien que, comme au début du XIX ${ }^{\mathrm{e}}$, les pirates font toujours fi des frontières et les témoignages indiquent que la Chine méridionale constitue un indéniable relais pour ce genre d'activité. Avec l'enregistrement des «kapal hantu» dans les pays «bienveillants» d'Amérique centrale et l'implication de banques asiatiques, la piraterie mari-

63. De son côté la police indonésienne a arrêté 35 pirates en juin/juillet 1992, à l'époque de la recrudescence des attaques dans l'archipel de Riau (International Maritime Bureau, Piracy Report 1992).

64. The Star, 29.8.97.

65. New Straits Times, 10.10.96.

66. The Star, 29.8.97. 
time est devenue aujourd'hui une branche d'activité de réseaux mondiaux, n'échappant pas ainsi au mouvement général de «globalisation».

$\mathrm{Si}$, dans l'archipel insulindien du début du XIX $\mathrm{X}^{\mathrm{e}}$ siècle, les pirates pouvaient bénéficier de la neutralité ou de la complicité des princes musulmans plus ou moins indépendants, aujourd'hui certains témoignages semblent montrer que les pirates bénéficient parfois de celle d'agents de douanes, de membre des polices maritimes ou encore de militaires de marines locales.

Comme il y a un siècle et demi où le premier effort pour lutter contre la piraterie a consisté à centraliser les informations sur les pirates à Batavia, la recrudescence récente a conduit à la mise en place en 1992 d'un centre régional de la piraterie à Kuala Lumpur, chargé de collecter toutes les informations non seulement pour l'Asie du Sud-Est mais au niveau mondial. Cette décision d'installer le centre en Malaisie confirme bien que la Méditerranée Sud-Est asiatique est aujourd'hui la région la plus touchée par la piraterie maritime. Parallèlement, l'effort important de cartographie qui a représenté un élément significatif dans la lutte au siècle dernier a fait place aujourd'hui à un effort non moins important dans le domaine de la détection, avec l'installation de puissants radars dans les zones sensibles comme le Détroit de Malacca. Aujourd'hui la lutte contre la piraterie s'opère également au moyen des missions conjointes de surveillance, mises en place notamment par certains pays de l'ASEAN, ou par la création d'unités d'intervention spécialisées. 